\title{
Enhancing sewage sludge dewaterability by a skeleton builder: biochar produced from sludge cake conditioned with rice husk flour \\ and $\mathrm{FeCl}_{3}$
}

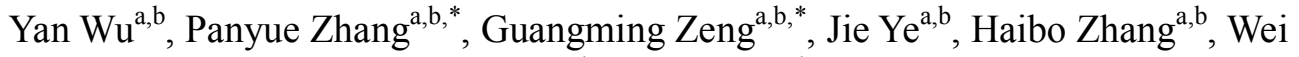
Fang $^{\mathrm{a}, \mathrm{b}}$, Jianbo Liu ${ }^{\mathrm{a}, \mathrm{b}}$

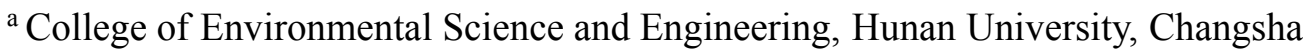
410082, People's Republic of China

${ }^{b}$ Key Laboratory of Environmental Biology and Pollution Control (Hunan University), Ministry of Education, Changsha 410082, People's Republic of China

E-mail address of all authors: wuyan19850827@hotmail.com (Yan Wu), zhangpanyue@hnu.edu.cn (Panyue Zhang), zgming@hnu.edu.cn (Guangming Zeng), eyejie@126.com (Jie Ye), haibo_zhang@hnu.edu.cn (Haibo Zhang), fw8905@163.com (Wei Fang), liujianbo901114@163.com (Jianbo Liu)

*Corresponding author:

*Panyue Zhang

E-mail: zhangpanyue@hnu.edu.cn

Tel.: +86 15001255497. Fax: +86 73188823701 .

*Guangming Zeng

E-mail: zgming@hnu.edu.cn

Tel.: +86 13908482238. Fax: +86 73188823701 .

Journal: ACS Sustainable Chemistry \& Engineering

Document prepared: Sept. 5, 2016

Total page number: 8

Total figure number: 4

Total table number: 2 


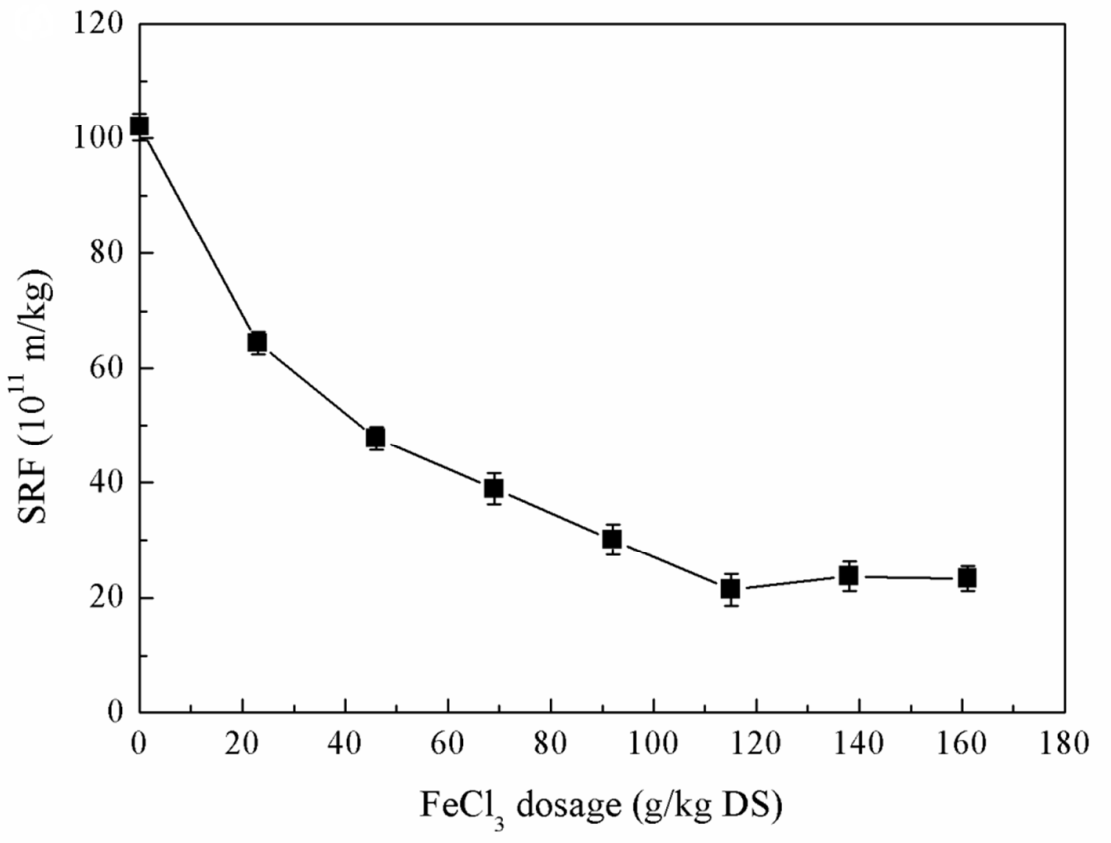

Figure S1 Effect of $\mathrm{FeCl}_{3}$ dosage on sludge dewaterability. 

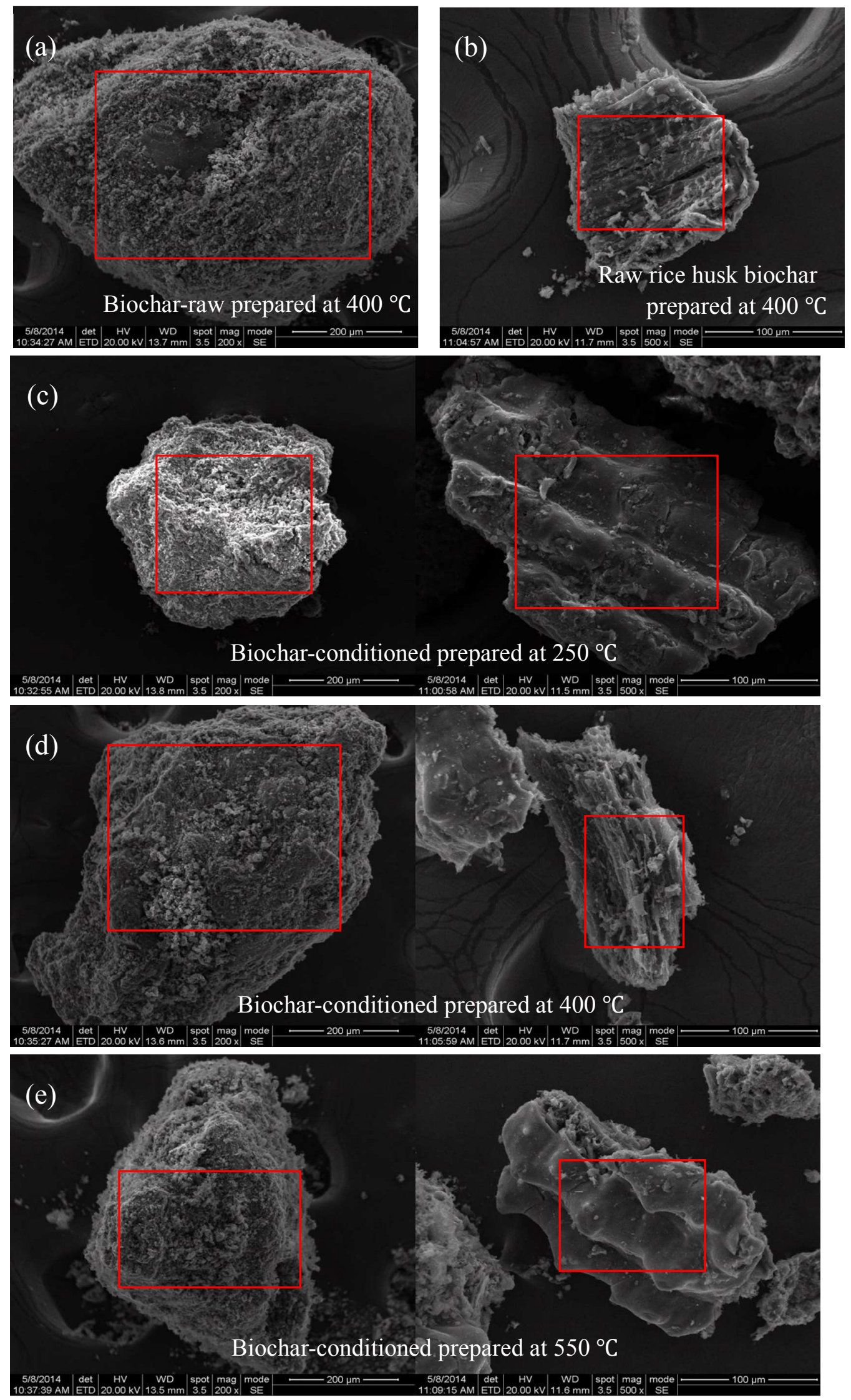


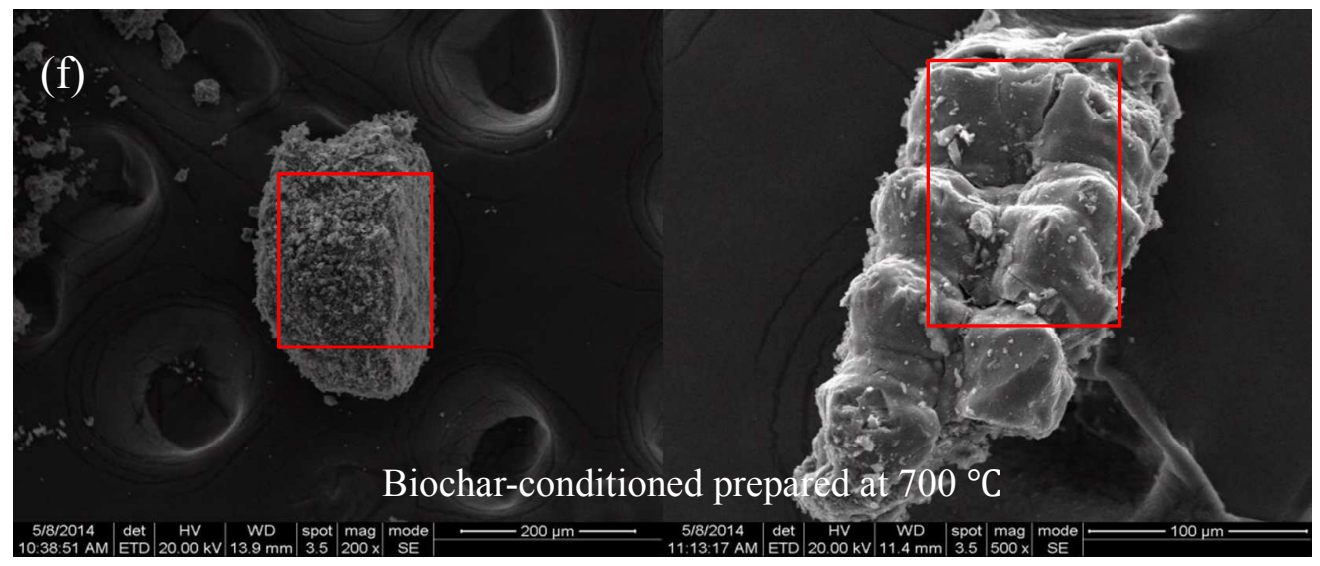

Figure S2 Effect of pyrolysis temperature on biochar-conditioned microstructure. (a) Biochar-raw prepared at $400{ }^{\circ} \mathrm{C}$, (b) raw rice husk biochar prepared at $400{ }^{\circ} \mathrm{C}$, (c) biochar-conditioned prepared at $250{ }^{\circ} \mathrm{C}$, (d) biochar-conditioned prepared at $400{ }^{\circ} \mathrm{C}$, (e) biochar-conditioned prepared at $550{ }^{\circ} \mathrm{C}$, (f) biochar-conditioned prepared at $700{ }^{\circ} \mathrm{C}$ (Scan area by EDS was in red box). 
(a)
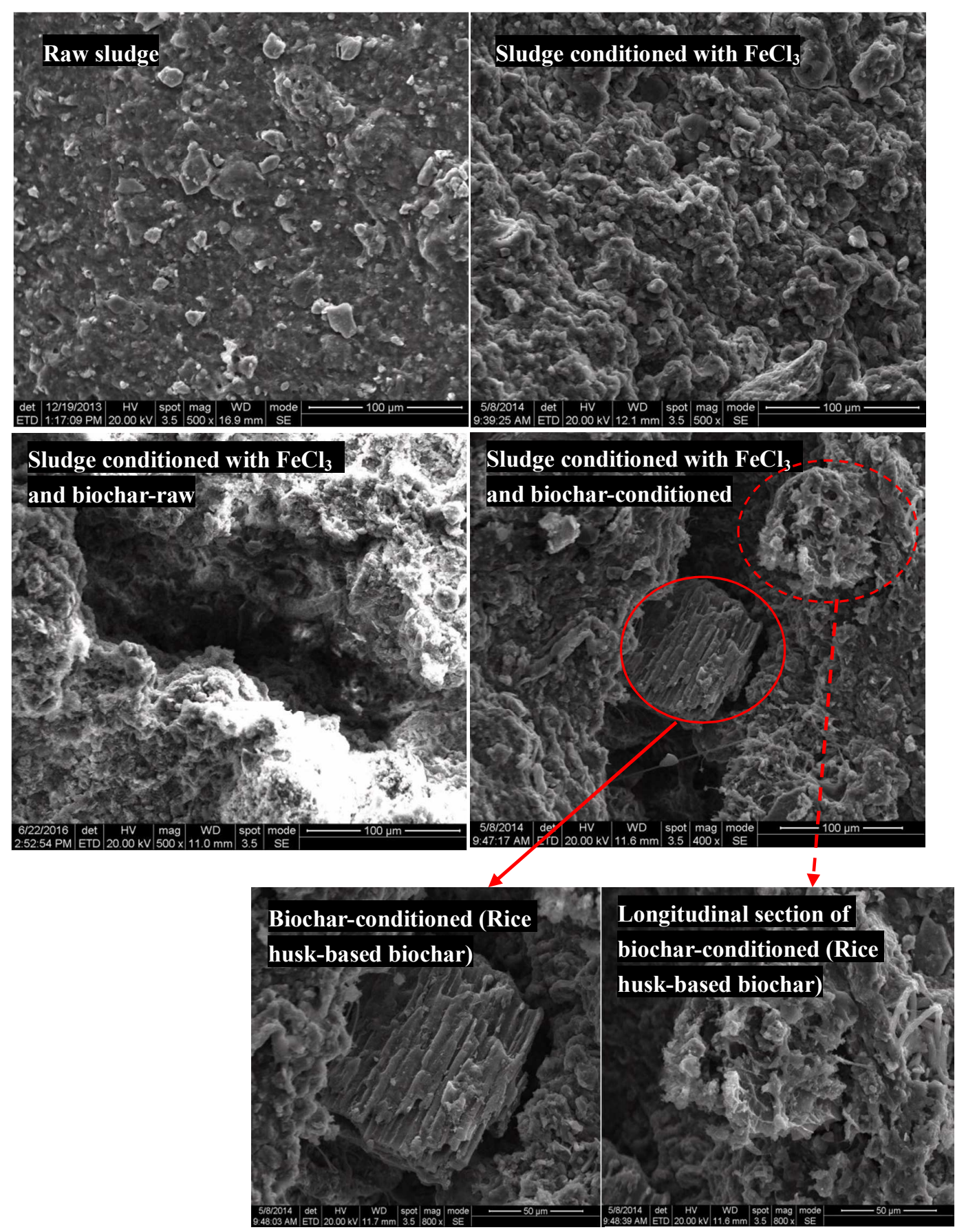
(b)

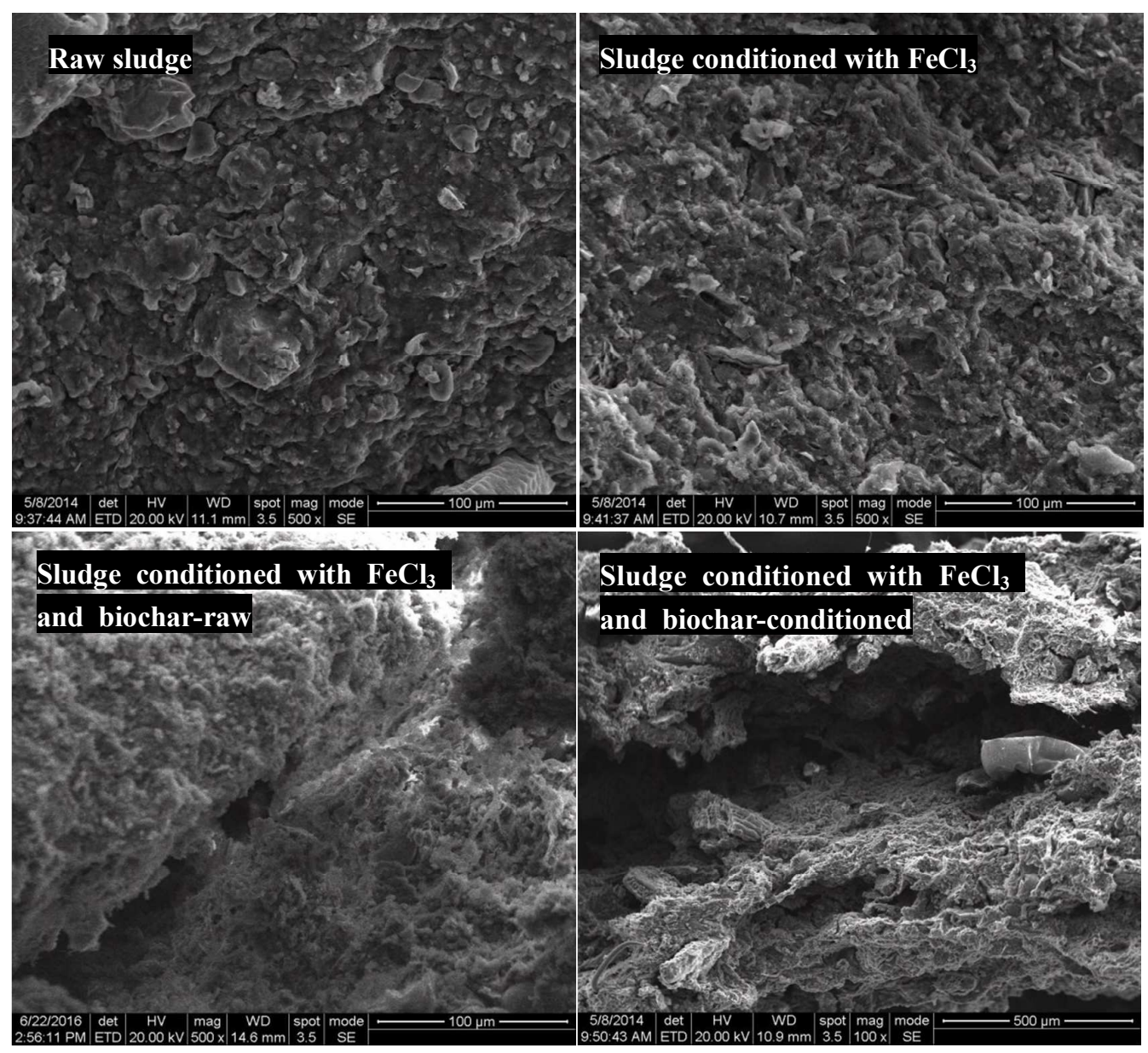

Figure S3 Change of microstructure of sludge cakes. (a) Surface of sludge cakes, (b) longitudinal section of sludge cakes $\left(\mathrm{FeCl}_{3}\right.$ dosage of $115.07 \mathrm{~g} / \mathrm{kg} \mathrm{DS}$, biochar-raw dosage of $40 \%$ DS, biochar-conditioned dosage of $70 \% \mathrm{DS})$. 


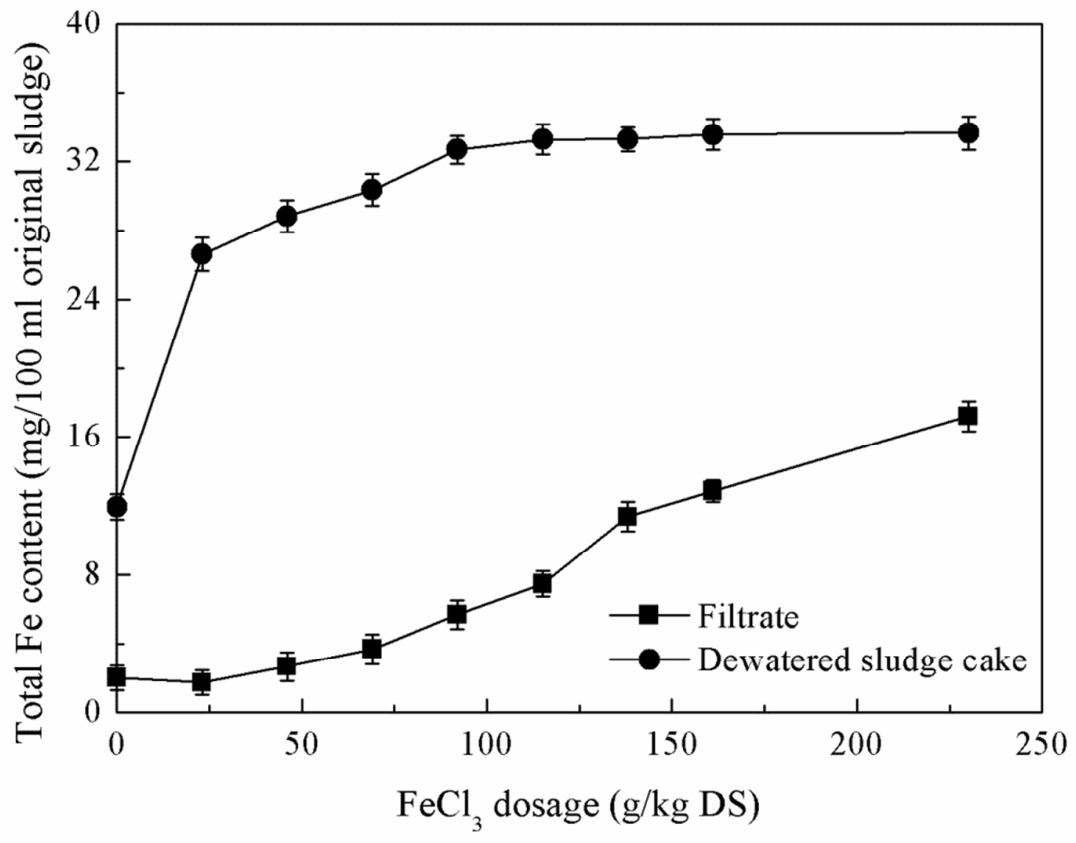

Figure S4 Effect of $\mathrm{FeCl}_{3}$ dosage on iron distribution in filtrate and dewatered sludge cake (Biochar-conditioned dosage of $70 \% \mathrm{DS}$ ). 
Table S1 Carbon content of biochar-conditioned surface tested by EDS (wt. \%) (Scan area marked in red box as shown in Supporting Information Figure S2).

\begin{tabular}{ccc}
\hline & $\begin{array}{c}\text { Sludge-based biochar } \\
\text { in biochar-conditioned }\end{array}$ & $\begin{array}{c}\text { Rice husk-based biochar } \\
\text { in biochar-conditioned }\end{array}$ \\
\hline $250^{\circ} \mathrm{C}$ & 30.08 & 60.56 \\
$400^{\circ} \mathrm{C}$ & 34.32 & 62.16 \\
$550^{\circ} \mathrm{C}$ & 42.75 & 69.29 \\
$700^{\circ} \mathrm{C}$ & 55.51 & 65.58 \\
\hline
\end{tabular}

Table S2 Iron content of biochar surface tested by EDS (wt. \%) (Pyrolysis temperature of $400{ }^{\circ} \mathrm{C}$, scan area marked in red box as shown in Supporting Information Figure S2).

\begin{tabular}{cc}
\hline & Iron content (wt. \%) \\
\hline Biochar-raw & 6.25 \\
Raw rice husk biochar & 0.35 \\
Sludge-based biochar in biochar-conditioned & 16.01 \\
Rice husk-based biochar in biochar-conditioned & 1.39 \\
\hline
\end{tabular}

